\title{
Germanica
}

allemande : fascination et suspicion

\section{Quand le juke-box aidait à vivre... Peter Handke et la musique : une approche}

Als die Jukebox geholfen hat... Einige Überlegungen zu Peter Handkes Verhältnis zur Musik.

\section{Brigitte Desbrière-Nicolas}

\section{(2) OpenEdition}

\section{Journals}

Édition électronique

URL : http://journals.openedition.org/germanica/1529

DOI : 10.4000/germanica.1529

ISSN : 2107-0784

\section{Éditeur}

Université de Lille

\section{Édition imprimée}

Date de publication : 30 juin 2005

Pagination : 163-180

ISBN : 9782913857155

ISSN : 0984-2632

Référence électronique

Brigitte Desbrière-Nicolas, « Quand le juke-box aidait à vivre... Peter Handke et la musique : une approche », Germanica [En ligne], 36 | 2005, mis en ligne le 13 juillet 2012, consulté le 06 octobre 2020. URL : http://journals.openedition.org/germanica/1529; DOI : https://doi.org/10.4000/germanica.1529

Ce document a été généré automatiquement le 6 octobre 2020.

(c) Tous droits réservés 


\title{
Quand le juke-box aidait à vivre... Peter Handke et la musique : une approche
}

\author{
Als die Jukebox geholfen hat... Einige Überlegungen zu Peter Handkes Verhältnis \\ zur Musik.
}

Brigitte Desbrière-Nicolas

\begin{abstract}
Et alors, les mélodies des Beatles, des Rolling Stones, des Creedence Clearwater Revival ? Elles m'ont « fait venir au jour » comme on met au jour un trésor ${ }^{1}$.

Peter Handke, Le Matin, à la fenêtre sur le rocher
\end{abstract}

1 À la fin de 1989, alors que les chaînes de télévision viennent de présenter au regard incrédule du monde l'effondrement du Mur de Berlin et qu'elles s'apprêtent à diffuser en boucle les images de l'exécution du couple Ceausescu, Peter Handke choisit quant à lui de se détourner de cette "année historique » (JB, 26) et de retenir dans le maillage de l'écriture un objet de la culture populaire en voie de disparition: le juke-box ${ }^{2}$. Ce texte fait partie d'une série de trois « essais $»^{3}$ dont le propos est précisé par l'écrivain dans une interview : «J'ai voulu écrire des petits livres qui seraient comme l'essence de la littérature $»^{4}$. Le choix générique affiché dans le titre par celui qui, quelques vingt ans plus tôt, déclarait être un "habitant de la Tour d'ivoire " dilatation du dire sur soi enchâssée dans une réflexion sur le travail d'écriture. Et c'est bien un portrait éclaté de l'écrivain que livrent au lecteur les trois essais. Alors que le premier ouvrage du cycle est une sorte de dialogue distancié avec un interlocuteur fictif remettant en perspective les expériences de l'enfance, et où le «je » s'impose, le livre sur le juke-box, consacré aux années d'adolescence, prend curieusement la forme d'un récit à la troisième personne. Plusieurs strates le constituent. Sous le prétexte de trouver un lieu propice à la réflexion sur cet objet banal et démodé, qui pourtant se pare d'étrangeté quand il surgit inopinément au premier plan dans l'œuvre de Peter 
Handke, l'écrivain décrit un voyage hivernal loin des bruits du monde dans les paysages arides du plateau castillan ${ }^{6}$ où s'inscrit la genèse de l'ouvrage. Nombreux sont les auteurs d'études relatives à ce texte qui fondent leur approche sur l'analyse des conditions de l'élaboration d'une écriture visant à retenir dans l'image l'éphémérité de

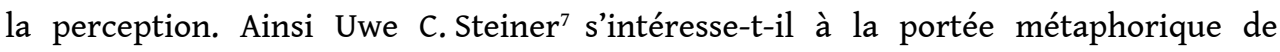
l'évocation d'un idéogramme tracé par la serveuse du restaurant chinois découvert par hasard à la fin du récit. Steiner démontre aussi tout le sens que prennent dans cette perspective dès le début du livre les observations apparemment anodines du voyageur à son arrivée à la gare routière de Burgos et les conclusions qu'il en tire :

Dans l'asphalte du quai, une grande tache de suie ; [...] la couche noire était épaisse, croisée par les diverses traces de semelles et de roues de valises [...] toutes ces empreintes de souliers..., c'était une image qu'il pouvait emporter pour la suite du voyage ${ }^{8}$.

2 L'image liée à cette perception visuelle que le voyageur solitaire décide d'emporter avec lui épuise-t-elle toute sa signification dans le contexte de la réflexion poétique ? Si l'on prend en considération le fait que l'observateur décide d'adjoindre aux marques déjà imprimées ses propres traces, il est permis de lire dans ce geste symbolique l'affirmation d'un désir d'appartenance à une communauté, fût-elle anonyme. Trouver sa place dans le monde, trouver sa place parmi les hommes est précisément le thème qui sillonne les récits de jeunesse décrivant l'errance d'individus en crise et où apparaît discrètement cette boîte à musique des temps modernes, le juke-box ${ }^{9}$.

3 Avant de résoudre l'énigme du juke-box, il est utile de s'interroger sur la perspective narrative qui caractérise l'essai dont il est l'objet. L'écrivain la justifie en exposant au milieu du livre (JB, 67-74) comment il a été amené à renoncer au projet initial d'un dialogue théâtral et à choisir le genre de l'essai, forme plus ouverte, propice à l'alternance d'images instantanées et de longues rétrospectives, et au passage abrupt de considérations générales à des évocations particulières. Cette forme offre pour P. Handke un avantage majeur : elle instaure une correspondance entre le mouvement de la réflexion et celui des expériences vécues associées au juke-box. L'évolution ultérieure de la forme de l'essai vers la narration est présentée comme le résultat d'une révélation: elle surgit du rythme d'une randonnée solitaire au cours de laquelle les perceptions du marcheur s'installent immédiatement en lui comme un récit écrit au passé dans lequel, grâce à l'imagination, le lieu traversé trouve sa place. Pourtant cet acquis ne tarde pas à subir les assauts du doute dans le dialogue imaginaire que le narrateur conduit avec lui-même: le choix ultime du récit ne relève-t-il pas de l'imposture, n'exprime-t-il pas une peur viscérale de la dispersion, de l'absence de cohérence? Ce choix peut ainsi être interprété comme le révélateur d'une crise profonde menaçant un individu qui se demande par ailleurs si les voyages entrepris au cours des dernières années, si l'existence nomade, ne sont pas en réalité une fuite sans retour (JB, 24), la recherche d'un ancrage hypothétique et provisoire, loin des bruits du monde. Dans ces circonstances, le juke-box devient par son étrangeté un objet de prédilection pour qui éprouve le besoin de fuir le monde (JB, 27).

4 Au-delà des justifications énoncées par le narrateur, il est possible d'établir par ailleurs une relation entre le récit à la troisième personne et la période remémorée, à savoir celle du passage de l'adolescence à l'âge adulte, celle de la rupture avec les appartenances imposées (familiales et sociales) et de l'affirmation délicate d'une appartenance choisie. La mise en œuvre de cette stratégie génère une distance qui à la fois protège le narrateur et stimule l'acuité de son regard. Le nouveau travail de 
mémoire qui s'engage, ce «WIEDER/HOLEN $»^{10}$, n'est pas une simple répétition, mais un retour opéré par l'écriture, une recherche fondatrice de continuité, qui interviennent au moment où $P$. Handke vit une nouvelle rupture en abandonnant la sédentarité et son superbe isolement sur le Mönchsberg à Salzbourg.

$5 \quad$ Avec l'Essai sur le juke-box le narrateur se propose d'éclaicir la question de savoir ce qu'a signifié cet appareil aux différents moments d'une vie maintenant bien avancée (JB, $11)^{11}$ et il ne manque pas de souligner la singularité de la démarche (JB, 33).

6 La mise en écriture du phénomène «juke-box » n'est pas nouvelle. Il est évoqué dans plusieurs récits du début des années soixante-dix. C'est incontestablement dans l'Angoisse du gardien de but au moment du penalty (1970) où elle est mentionnée à dixsept reprises que cette machine sonore, constamment désignée sous le terme de " musicbox », joue un rôle de premier plan. Elle est d'abord simplement un élément du décor des auberges-refuges marquant les étapes de l'errance du protagoniste qui, en proie à des troubles croissants de nature schizophrénique, devient meurtrier. Le jukebox est en outre un recours aux heures de solitude ou dans les moments de malaise croissant, lorsque l'étau de l'enquête policière se resserre. Sa seule présence permet de donner une contenance, voire d'apaiser temporairement les désordres intérieurs de celui que fascine l'automatisme de son fonctionnement. La précision du geste: l'introduction d'une pièce, la pression sur une touche, parfois même à la manière d'un automate, constitue l'élément prépondérant de l'évocation des rapports entretenus avec un appareil qui fait oublier les aléas des relations humaines. Il n'est pas question de musique dans ce livre mais toujours du mouvement impeccablement réglé du disque sélectionné, ainsi que de la faible lumière dispensée dans la pénombre. La reprise de ces traits caractéristiques dans Le recommencement (1986), qui est la relation d'un voyage initiatique dans le Karst, témoigne de leur importance. Le juke-box peut occasionnellement aussi être le lieu d'une rencontre éphémère et provisoirement salvatrice entre deux solitudes, par l'échange de quelques paroles, par la fugitive convergence des regards, par la danse ${ }^{12}$.

7 Si dans le récit de 1970 le juke-box se révèle être avant tout, par le biais de la fiction, un lieu-refuge, une présence lumineuse, un reflet de la quête nostalgique de relations intersubjectives non problématiques, l'essai de 1989 se fait plus explicite, il expose par touches dispersées ce qui confère à l'objet une dimension véritablement existentielle au cours de l'adolescence. Même si des considérations relevant de l'étymologie, de la typologie et de l'historique, concernant un appareil apparu aux États-Unis à l'époque de la prohibition, ne sont pas absentes, ces passages obligés s'effacent bien vite derrière les enjeux personnels suggérés cette fois encore par la reprise lapidaire d'éléments descriptifs récurrents déjà cités et relevant de la simple perception visuelle : la fascination exercée dans l'obscurité par la lumière artificielle et colorée de l'appareil en position de veille et par l'automatisme du fonctionnement des cylindres. En outre, une grande attention est portée aux lieux d'installation du juke-box ; perçu comme un point d'ancrage fragile pour les individus solitaires ou déracinés (émigrés ou soldats), il est implanté à la périphérie des métropoles touristiques, dans les régions frontalières, dans des espaces intermédiaires situés à l'écart d'une gare, d'un canal... Ce lieu est un endroit d'exception qui suscite chez le narrateur une sensation semblable à celle 
éprouvée dans l'enfance à l'intérieur des greniers à foin dispersés dans les prairies, celle d'avoir trouvé sa place, d'être arrivé là où l'on se sent à l'abri $(\mathrm{JB}, 65,78)$. Là où l'on échappe aux pesanteurs intérieures que l'auteur retrouve chez certains caractères de Théophraste médités pendant le voyage : êtres solitaires, mal à l'aise dans la société de leur temps, ils simulent une appartenance factice et ridicule avec l'énergie du désespoir (JB, 124). Dès le début du séjour dans les paysages arides de Castille, le jukebox est en effet désigné comme un objet qui exerce une attirance particulière sur les individus en situation de rupture avec le monde (JB, 27). Dans l'essai, la perception sensorielle devient plus complexe et dépasse le registre du visuel. Les souvenirs d'enfance et d'adolescence attribuent à la sonorité particulière provenant des entrailles de l'appareil la capacité de plonger l'auditeur dans une atmosphère de recueillement (JB, 79). De cette caisse de résonance insolite s'élèvent les voix et les mélodies venues d'un ailleurs auréolé de prestige, qui n'a rien perdu de la force d'attraction exercée sur des aînés illustres dans les années vingt. La vibration étrange des chants de détresse, d'espoir et d'amour se dilate et se transmet à l'individu en recherche de lui-même. Contrairement à l'auteur des œuvres de fiction, l'écrivain-essayiste met l'accent sur le type de musique diffusée, ces mélodies américaines à la mode, qui remplissent de joie, de chaleur et d'un sentiment d'appartenance communautaire le lycéen pauvre de Carinthie : le regard porté sur la touche commandant Memphis, Tennessee est déjà la promesse d'un départ et d'une transformation mystérieuse de l'être (JB, 84). L'écoute attentive ne détourne pas de soi, ne transporte pas dans un monde rêvé mais elle engage un processus de concentration qui apaise, éveille aux possibles et fortifie la foi en l'avenir ${ }^{13}$. L'expérience singulière, un soir d'hiver, dans un des "cafés à juke-box " fréquenté quelques années plus tard par l'étudiant solitaire, est à cet égard très significative (JB, 87-90). Le jaillissement inopiné des profondeurs de la machine d'une chanson non choisie, interprétée par un groupe inconnu «choeur insolent d'anges chanteurs » (JB, 89) est l'agent d'un prodige, d'une naissance à soi et au monde ${ }^{14}$ :

Une musique se mit à jaillir des profondeurs qui pour la première fois de la vie, plus tard cela n'arriva plus qu'aux instants de l'amour, lui apprit ce que dans une langue spécialisée on appelle « lévitation » et qu'il lui fallait appeler, comment donc?, un quart de siècle plus tard : «ascension »?, « abolition des limites »?, « expansion au monde entier » ? Ou bien : «Cela, cette mélodie, ce son - c'est moi, là, maintenant ; avec ces voix, ces harmonies je suis devenu, comme jamais encore, celui que je suis $^{15}$.

8 Toutefois l'auteur de l'essai ne se contente pas de faire œuvre de mémoire et d'analyser la signification de cette boîte à musique qui a été pour l'adolescent génératrice d'émancipation, il médite aussi sur les attentes présentes du narrateur presque quinquagénaire. La forme du juke-box et la résonance particulière ne sont plus déterminantes. En revanche, la liste des titres revêt une importance singulière. Elle doit échapper à la standardisation d'une programmation mercantile et refléter par la diversité des titres, des styles et des écritures, la dimension d'imprévu recelée par cette merveilleuse machine. Ce n'est pas quand il est désœuvré que l'écrivain fréquente dans son pays d'origine les établissements dotés d'un juke-box, mais après les longues heures consacrées à l'écriture. La recherche du juke-box fait alors pendant à l'activité de la marche qui installe le silence préalable au travail effectué dans une maison sans musique. Cette quête débouche sur une écoute lacunaire du juke-box qui ne répond pas à un banal besoin de distraction après la fatigue du jour. Elle ne vise plus comme par le passé à procurer une concentration et un recueillement qui sont ouverture aux possibles. Dans le présent de la narration, elle permet, accompagnée par la perception 
simultanée du cadre environnemental extérieur, de générer une présence au monde renouvelée. Condition indispensable à la poursuite de l'œuvre en cours d'élaboration, l'audition des mélodies diffusées par le juke-box redonne vie et contours aux images assoupies au fond de l'être (JB, 99-103).

9 À l'évidence, le juke-box n'est pas en 1989 pour Peter Handke un accessoire pittoresque exhumé des années d'adolescence et qui «aidait à vivre». L'écrivain reconnu le considère toujours comme une sorte de catalyseur, comme un facteur déclencheur de processus aux enjeux complexes, mais il accorde cette fois un rôle accru à la composante musicale, vocale et instrumentale, qui s'affirme d'ailleurs dès le récit du passage à la trentième année, par le biais de la relation d'un périple à travers les ÉtatsUnis sur fond de crise conjugale : La Courte lettre pour un long adieu (1972).

Quand sur un air de scandale, Peter Handke, jeune étudiant en droit, fait brutalement irruption sur la scène littéraire allemande par ses invectives lors de la réunion annuelle du Groupe 47 à Princeton en 1966, il est pour la presse le «Beatle de Graz », en raison de sa coiffure. Mais en 1972 la « pop star de la littérature » a pris ses distances avec les expérimentations formelles radicales et la provocation brutale. C'est alors qu'apparaissent tant dans la fiction américaine déjà citée que dans le livre à coloration autobiographique consacré au suicide de sa mère, Le Malheur indifférent ${ }^{16}$, des titres ou des citations illustrant ses choix musicaux. Une lecture croisée de ces deux œuvres et de l'Essai sur le juke-box révèle en filigrane l'apparition précoce de thèmes dont les imbrications étroites et la permanence s'affirmeront ultérieurement, à savoir la dimension émancipatrice de la musique vocale pour l'individu et ses implications dans la réflexion du poète sur l'écriture.

11 La nécessité du départ, de la prise de distance salvatrice vis-à-vis de la terre des origines est progressivement manifestée dans l'essai au travers du mot Aufbruch. Ardemment désiré par le jeune homme (JB, 84), cet Aufbruch est ressenti par le narrateur comme accompli au terme du voyage (JB, 139), sans que soient clairement distinguées la part prise par la décontextualisation géographique et celle relevant du travail de mémoire et de réflexion attaché au juke-box. L'essai résonne à ce propos comme un lointain écho de la quête nostalgique du protagoniste de La Courte lettre qui a fui son Autriche natale et attend dans la fièvre son trentième anniversaire. Le nouveau monde, où il a trouvé temporairement refuge, doit le libérer du harcèlement incessant et paralysant d'une peur générée par les traumatismes d'une enfance pauvre, vécue dans un environnement humain et socio-culturel étriqué caractérisé par l'austérité, les contraintes, les rites, les routines et l'effacement de l'individualité. La crise profonde provoquée la même année par la mort volontaire de la mère de l'écrivain, conduit Peter Handke à dépasser la fiction pour faire un retour sur sa propre enfance et à brosser - à l'arrière-plan du destin individuel de la femme - un tableau impitoyable de la Carinthie dont sa mère et lui-même sont issus.

12 Il peut à première vue paraître étonnant que, dans Le Malheur indifférent, le premier exergue en langue anglaise soit emprunté à une chanson de Bob Dylan («Its Alright, $\mathrm{Ma} / \mathrm{I}$ 'm Only Bleeding ») : «He not busy being born is busy dying ». Comme l'a montré Cecile Cazort Zorach ${ }^{17}$, il ne s'agit pas ici tant de souligner les liens unissant le fils et la mère et d'illustrer le vide d'une existence que de suggérer au moyen des citations 
étrangères l'espoir recelé par un langage libéré des entraves. Mode d'expression figé dictant les comportements, le langage a été pour la mère un agent d'oppression, tuant toute individualité. Dans ces circonstances, il importe au fils écrivain-biographe de restituer au langage, par l'introduction d'un élément culturel extérieur, sa fonction de communication et sa force de libération. Ce n'est pas un hasard si la mère tente vainement à deux reprises de mener une existence nouvelle au-delà des frontières (en Allemagne !) puis commence timidement à parler d'elle-même après avoir lu avec son fils des romans étrangers. Mais le seul départ (Aufbruch) auquel elle accédera, sera l'extinction définitive dans la mort par l'absorption de barbituriques. Si la mention de Bob Dylan est associée au rêve de la libération du carcan d'origine socio-culturelle, il faut bien admettre que l'aspect musical semble cette fois encore pour le moins secondaire! Cependant l'allusion voilée à Paul Simon dans les notations éparses de la fin inachevée du livre conduisent à nuancer ce jugement: "J'ai souvent senti en écrivant cette histoire qu'il serait plus conforme aux faits d'écrire de la musique, Sweet New England... $»^{18}$. Néanmoins il faut remarquer avec Cecile Cazort Zorach que le choix ne concerne pas la musique en tant que telle, une musique "non verbale", mais une musique vocale populaire, le folk song, auquel Peter Handke associe dans une chronique du Spiegel ${ }^{19}$ 'écriture de Patricia Highsmith, l'auteur du second exergue, dont les textes seraient mystérieusement éclairants comme les récits dans les chansons de Paul Simon.

La narration, forme d'écriture glorifiée dans le livre de 1986 Le Recommencement, forme de la communication par excellence, forme de l'adresse clarificatrice à l'autre, le témoin privilégié, explique l'engouement précoce de l'écrivain pour des types de musique nés dans les tréfonds de l'Amérique ou de la société pré-industrielle anglaise et trouvant leur origine dans les chants d'une humanité laborieuse, voire souffrante. Si l'on cite pêle-mêle des noms d'auteurs interprètes légendaires (Bob Dylan, Van Morrison, Otis Redding, John Fogerty), de groupes renommés (Canned Heat, Creedance Clearwater Revival, Rolling Stones) et des titres évocateurs égrenés dans les livres de 1972 et dans l'Essai sur le juke-box (On The Road again, Route sixty-six, Redemption...), l'on constate que tous conduisent selon des voies plus ou moins détournées au blues, au folk song et aux courants protéiformes qui en sont issus, qui s'y sont mêlés, s'en sont éloignés et aux hommes qui, au fil des décennies, y ont puisé une inspiration originale. Cette musique, l'écrivain ne l'aborde pas à la façon des spécialistes soucieux de recherche et d'archivage de la musique populaire de l'entre-deux-guerres. Elle apparait dans le cadre d'expériences directes du jeune homme, amateur de juke-box et à un moindre degré de radio, qui partage sans tapage la fièvre qui a saisi sa génération quand elle a découvert la magie du blues. Wim Wenders, collaborateur occasionnel de Peter Handke, la définit ainsi : « [c'était] une manière d'aimer l'Amérique sans se laisser avoir par l'impérialisme et la corruption. Le blues était subversif. Radical. Existentialiste. [...] Il venait des marginaux, des rebelles de la société américaine, il avait une qualité unique, brute et rocailleuse... $»^{20}$. Il est permis de considérer que l'engouement de Peter Handke pour cette musique n'est pas uniquement imputable à un phénomène de mode déferlant sur l'Europe dans les années soixante. Tout porte à croire que l'universalisme thématique du blues et des formes dérivées, que l'expression répétée dans le chant des peurs, des frustrations de l'interprète, de ses espoirs ou de sa résignation, de son désir de mise en route vers un monde meilleur, a constitué pour l'étudiant en quête d'identité, de reconnaissance et de libération un véritable point d'ancrage qui était promesse de départ. Le blues inscrit la solitude dans l'universel et 
assure la survie. Jim Dickinson le déclare : « le blues n'est pas la musique de l'esclavage, c'est celle de l'émancipation ${ }^{21}$. Le blues est un sentiment de soi qui se fait chant. Peignant les événements de la vie quotidienne, il est accessible à tous. La primauté initiale de la parole en fait une forme d'art privilégiée pour Peter Handke. En effet, c'est sur le langage que la substance mélodique prend appui. Un épisode de La Courte lettre illustre de façon saisissante la force d'exorcisation exercée par un chant disant l'insoutenable souffrance. Désireux d'échapper à l'emprise de la solitude et à son cortège de maléfices, le protagoniste se rend dans un night-bar où se produit un chanteur qui s'accompagne seul à la guitare. Le silence se fait quand il raconte son histoire, celle d'un homme issu du viol d'une faible d'esprit par un fermier. Élevé comme le fils du couple de ce dernier, il est un jour délivré par la jeune muette qui lui a donné le jour, des fils d'une clôture qu'il avait entrepris d'escalader. Et l'enfant dit à la femme du fermier : " "Hé, Mère, pourquoi l'idiote a-t-elle des mains si douces ? et cette idiote c'était ma mère !" criait le chanteur. Il leva sa guitare, se ramassa sur lui-même et commença à jouer de longs accords aux vibrations prolongées ${ }^{22}$. Et tout se passe alors comme si ce récit dépouillé et douloureux et les vibrations musicales qui lui succèdent sur le mode paradoxal d'une méditation silencieuse, déclenchait chez un auditeur, ancien soldat du Vietnam rencontré quelques heures plus tôt par le protagoniste, un état de transe au moment où « la musique se faisait plus tranchante, plus impatiente » :

... et pourtant je le contemplais avec respect et sympathie. Sa transe n'était pas artificielle... elle lui était tombée dessus et il ne savait qu'en faire. Il ne savait plus parler, pas même bredouiller, aussi pour se délivrer se comportait-il comme si un très ancien monstre mourait en lui ${ }^{23}$.

14 Ne s'agit-il que d'un passage anecdotique, commenté très laconiquement par le voyageur, ou n'est-ce pas plutôt la représentation imagée d'une nostalgie lancinante de délivrance proposée à la sagacité du lecteur?

Un groupe en vogue dans les années soixante au nom interminable et alambiqué mérite dans ce contexte de retenir l'attention ${ }^{24}$ : les Creedence Clearwater Revival. Un jeu de collage lui confère une valeur programmatique et, pour l'écrivain autrichien, sans doute aussi une portée symbolique : foi et confiance sont suggérées par l'association hasardeuse dont résulte le premier terme, authenticité et pureté transparaissent ensuite dans une formule publicitaire pour une marque de bière, le tout débouchant sur l'espoir d'un renouveau.

Mais quels qu'aient été l'enthousiasme et les attentes de l'adolescent, puis de l'étudiant, il ne lui est pas aisé de se détacher de traditions musicales omniprésentes dans un pays comme l'Autriche. L'auteur de l'essai se remémore la mauvaise conscience qui l'a envahi face à son engouement pour cette « non-musique » (JB, 83); ce malaise insidieux et durable le conduit, lors de l'achat de ses premiers disques, à se tourner vers ce qui était communément reçu comme « musique ».

D'ailleurs les allusions à des formes musicales consacrées, à divers titres, ne manquent pas dans le récit relatant la vie de la mère et l'intention critique ne saurait échapper au lecteur attentif. On y distingue une musique rejetée par l'auteur, celle des marches militaires qui accompagnent l'entrée d'Hitler à Klagenfurt et les communiqués de victoire pendant la guerre, celle des concerts de musique wagnérienne à la radio et la musique légère, diffusée la nuit par l'émetteur du Reich à Königsberg. Toutes tendent en effet à réjouir les cœurs et elles donnent à la fois le sentiment illusoire d'exister et 
celui d'une appartenance communautaire élargie mais toutes sont des agents sournois de manipulation et de dépossession de soi. Plus anodine est à première vue la pratique musicale du chant choral, prisée en Autriche, activité à laquelle s'adonne la protagoniste, jeune fille gaie et gourmande de la vie. Mais dans un ordre social rigide et austère qui bannit toute velléité d'individualisme, elle apparaît davantage comme un exutoire destiné à canaliser les trop-pleins d'énergie et à contenir les aspirations secrètes à l'autonomie ${ }^{25}$. Quant aux chansons à succès, qu'elles relèvent du folklore ou des variétés de l'époque, le simple fait de les fredonner procure un allégement éphémère dans un quotidien constitué de travail et de misère (WU, 21-22, 26, 18, 37, 43). Une indubitable amertume émane enfin de l'évocation fugitive du concert radiophonique à la carte. Alors que la détresse, la solitude, la dépression s'abattent irrémédiablement sur la mère du fils-narrateur, des parents de Berlin-Est dédient à la famille en voie de décomposition l'Alléluia de Haendel! Comment évoquer avec une ironie plus mordante le décalage radical existant entre la réalité des uns et des autres et les sphères éthérées d'une musique qui est devenue le simulacre institutionnalisé de l'harmonie et de la joie. Parler de haine de la musique à la façon de Pascal Quignard en ce qui concerne le jeune Handke des années soixante-dix serait abusif ${ }^{26}$, ne serait-ce qu'en raison de la concision et de la dispersion des rares notations relatives à ce sujet. Néanmoins l'attitude générale adoptée face au phénomène musical ambiant conduit à discerner dans Le Malheur indifférent des points de convergence avec les propos tenus par l'écrivain français au sujet de l'utilisation qui a été faite de la musique dans les camps nazis. P. Quignard dénonce d'une façon générale la fascination exercée par les rythmes musicaux sur les rythmes corporels et, se référant à Primo Levi, il qualifie la musique de "maléfice» et vilipende "l'hypnose du rythme continu qui annihile la pensée et endort la douleur ». Et d'ajouter que sa mise en œuvre sert à augmenter l'obéissance et « à souder tous les individus dans la fusion non personnelle, non privée, qu'engendre toute musique ». À la différence de P. Quignard, P. Handke ne condamne pas indifféremment toute forme de musique. Sans doute accorderait-il quant à lui une interprétation positive au mot d'un compositeur selon lequel « son écriture comme son audition entraîne un désir de choses inexistantes", un désir, pour P. Handke, susceptible d'ouvrir les voies de la vie.

La Femme gauchère dans ses deux versions narrative et filmique, permet d'affiner la compréhension des rapports que l'écrivain autrichien entretient avec la musique. Si la biographie de la mère se présente comme une œuvre inachevée, il n'en est pas de même pour l'œuvre qui en constitue le pendant idéalisé, La Femme gauchère (1976) ${ }^{27}$. Die linkshändige Frau emprunte son titre à une chanson de Jimmy Reed ${ }^{28}$, chanteur de country-blues: The Left Handed Woman (1964). Marianne, la protagoniste dite gauchère, la diffuse en boucle au cours d'une longue nuit de solitude (lF, 101-102) après avoir mis brutalement un terme à sa vie conjugale à la suite d'une illumination, et s'être engagée en compagnie de son fils sur la voie semée d'écueils de la quête de soi et de relations renouvelées à l'altérité. L'originalité de la référence explicite à ce titre du répertoire du country-blues réside dans le fait que pour la première fois un texte de chanson y est adjoint (en langue allemande) et que cet ajout dépasse le cadre d'une technique d'écriture courante chez Handke, à savoir l'inscription d'un destin fictif dans un jeu de 
miroirs réfléchissant d'autres textes. En effet, le travail comparatif de Russel E. Brown relatif aux gauchères de Jimmy Reed et de Peter Handke ${ }^{29}$ fait apparaître que la version allemande des paroles - contrairement aux allégations de Manfred Durzak - s'avère n'être en rien une traduction, mais une création originale de l'écrivain autrichien, apposée au titre bien réel cité en anglais. Cela indique clairement que les diverses références musicales relevées dans les œuvres de $\mathrm{P}$. Handke doivent être considérées avec prudence et, dans une large mesure, être interprétées comme des éléments symboliques destinés à servir la fiction, sans que leur soit pour autant déniée une dimension émancipatrice. Russel E. Brown montre que le personnage de Marianne diverge en tout point des deux partenaires féminines d'une chanson qui ne correspond nullement à la situation de l'énigmatique protagoniste handkéenne en rupture d'appartenance. Seul le titre a été retenu en raison de son poids métaphorique soulignant la singularité du personnage, souvent interprété comme projection féminine de la subjectivité et de la marginalité de son auteur, lui aussi séparé de son conjoint et vivant avec sa fille. Au-delà des remarques de Russel E. Brown, il convient d'ajouter que l'écoute répétée du disque américain dans le récit, ressentie comme un besoin vital, confirme les observations déjà formulées, à savoir qu'elle sert d'élément de contraste, qu'elle s'oppose à une pseudo-écoute machinale de musique uniquement destinée à meubler superficiellement le vide intérieur passager ou celui de la conversation (IF, 13, 51-52). La chanson dans laquelle Marienne enveloppe les tourments de sa mise en route solitaire vers un avenir incertain, exprime dans la strophe finale le rêve étrange d'un amour absolu né de l'intensité du regard échangé, un motif récurrent qui s'épanouit dans le Don Juan (raconté par lui-même) de 2004 ${ }^{30}$ :

\footnotetext{
J'aimerais te voir DANS UN CONTINENT INCONNU

Car je t'y verrai seule enfin parmi mille autres

Et tu me verras MOI seul entre mille autres

Et nous irons enfin l'un à la rencontre de l'autre ${ }^{31}$.
}

Ce qui confère à La Femme gauchère une place particulière dans l'œuvre de Peter Handke, c'est l'acuité du regard que l'auteur porte sur son héroïne. Une approche comparative du récit et de la version filmique, effectuée sous l'angle de la composante musicale, permet de le mettre au jour. Présenté au Festival de Cannes en 1978, le film de P. Handke réalisé avec les encouragements de Wim Wenders, a été conçu pour répondre à une nécessité intérieure, celle d'inventer une femme mythique qui, malgré sa lassitude, se donne les moyens de vivre une expérience unique qui apportera l'apaisement et permettra de goûter des instants d'éternité32. Une métamorphose insolite s'opère entre l'œuvre narrative et sa mise en images. L'aventure de la gauchère, incarnée par Edith Clever, se dépouille de sa gangue de banalité manifestée non seulement par les références à la culture américaine dominante, par la chanson nostalgique du country-blues, mais aussi par l'histoire insipide d'une émancipation teintée de féminisme que Marianne doit traduire à titre professionnel. Le cinéaste lui substitue le célèbre conte de Flaubert (Un cœur simple) et la destinée morose et besogneuse de la servante Félicité sert désormais de toile de fond contrastée à la quête de Marianne. Par ailleurs, il serait sans doute provocateur mais tout à fait justifié de ne pas tant parler d'une mise en images du cheminement solitaire et finalement rayonnant de Marianne que d'une lente mise en silences de son aventure, d'une mise en silences s'insinuant résolument dans l'univers sonore du quotidien urbain, de la vie domestique, de la nature, des silences dont les intervalles sont fugitivement ponctués de musique baroque. 
20 La Femme gauchère n'est pas un film muet même si quelques scènes muettes le jalonnent, même si la part prise par la parole en générale est limitée et que la voix de Marianne ne se fait entendre que tardivement et parcimonieusement. Bien que la lenteur, le dépouillement des images et le regard ardent d'Edith Clever retiennent en premier lieu l'attention du spectateur, les éléments dominants s'avèrent être les nombreux espaces de silence, une cinquantaine dans le film (durée: une heure quarante-neuf minutes). Le silence peut être absolu, parfois il est entrecoupé de brefs échanges verbaux, il est déchiré par les agressions sonores urbaines ${ }^{33}$, il est scandé par le bruit des pas des protagonistes, il vibre aussi aux pulsations du quotidien que la surdité moderne réduit à l'inexistence : le frôlement d'une étoffe, le crépitement de la flamme... Il est aussi le réceptacle des voix de la nature, du chant des oiseaux, du craquement des feuilles foulées... Le silence n'est pas un vide absolu, il recueille les traces infimes de la vie et toute sa densité mystérieuse éclate quand la musique instrumentale vient le féconder.

21 "Musique de J. S. Bach» indique laconiquement le générique qui défile sur fond de mélodie exécutée à la flûte des Andes. La mention du seul nom du compositeur a-t-elle pour unique objectif d'accentuer, s'il en était besoin, l'étrange solennité qui auréole l'expérience inédite de Marianne et de souligner le décalage entre le récit filmique et l'environnement contemporain sur les images duquel celui-ci s'achève, des couloirs de métro où défilent des individus anonymes vus de dos. La teneur du message est quant à elle signalée en surimpression par une phrase extraite d'un poème de Vlado Kristl : « N'avez-vous donc pas remarqué qu'il n'y a de place que pour qui apporte sa propre place $»$.

Les notes égrenées, la plupart du temps en sourdine en une petite dizaine de séquences variant de douze à soixante secondes, sont empruntées au Prélude de la Suite $n^{\circ} 1$ en sol majeur pour violoncelle (BWV 1007). Interprétées à sept reprises à la guitare sèche, elles accompagnent dès la septième minute du film des transitions: les passages dans l'espace, entre intérieur et extérieur, les mouvances de l'intériorité à des moments décisifs de rencontre avec la solitude ou l'altérité familière (époux, amie, père) ou une altérité qui permet d'établir un intervalle entre ce qui emprisonne et ce qui est l'objet de la nostalgie (la destinée de la servante de Flaubert, proposée à la traduction par l'éditeur). Mais c'est incontestablement quand le violoncelle inscrit plus longuement à trois reprises dans le silence le Prélude de la Suite $n^{\circ} 1$, pendant le second mouvement du film (phase de résistance aux tourments de la solitude), qu'émane « la plus chatoyante poésie », pour citer le commentaire de Pablo Casals à propos des Suites pour violoncelle de J.S. Bach. Peut-être P. Handke a-t-il, comme le violoncelliste virtuose, été sensible à l'optimisme qui se dégage de la Suite $n^{\circ} 1$. Tandis que le chant de l'instrument veille dans une première scène nocturne sur l'héroïne endormie à sa table de travail et accompagne des images oniriques énigmatiques, il enveloppe dans deux séquences crépusculaires de longs instants de repli sur soi, puis de méditation à côté de l'enfant et assure dans une phase ultime le passage à la lumière d'une claire journée de mars où la mère et le fils s'engagent dans une longue marche à travers les espaces boisés des collines environnantes. Les autres éléments musicaux épousent les états d'être de Marianne: des images somptueuses et un morceau orchestral classique manifestent le sentiment de plénitude sereine éprouvé au cours d'un repas pris avec Stéphane, à l'oriental devant l'âtre, dans une sorte de cérémonial où les fruits exotiques éclatent, ouverts à la force des doigts... Les sonorités d'un piano invisible interprétant La Lettre à 
Élise s'insinuent dans les rues et ruelles désertes d'une ville de la banlieue parisienne où, par une marche "forcée", l'héroïne exorcise les tensions insoutenables qui l'accablent ${ }^{34}$. Un peu plus tard, une autre marche solitaire effectuée dans les bruits de la ville s'achèvera dans une église éclairée par un vitrail modeste mais lumineux, dans laquelle résonne une musique d'orgue. Mais quand l'apaisement s'installe subrepticement au bout de quelques semaines, quand le rayonnement de la gauchère a réussi à imposer un nouveau mode de communication avec l'altérité, la musique sonore s'éclipse, le silence de la musique s'installe. Il introduit une métamorphose des individus qui se dépouillent progressivement des rôles sociaux et des masques imposés ${ }^{35}$.

Dans la recherche relative à la place occupée par la musique dans les œuvres abordant le sujet de la quête identitaire, La Femme gauchère marque un tournant révélé par le passage du récit au film. Le destinataire de la production cinématographique n'est plus un interlocuteur solitaire mais un individu invité à partager dans une salle obscure, au milieu d'un groupe anonyme, une expérience esthétique requérant une dilatation, une diversification de la stratégie narrative. Elle bannit le primat de la communication verbale et ne se contente pas de proposer un regard insolite sur l'altérité, les relations intersubjectives et le quotidien urbain, elle donne à entendre un éventail élargi des bruits du monde, enchâssé dans la solennité du silence et le dépouillement d'une musique instrumentale non contemporaine qui instaurent une relation nouvelle entre l'intériorité et le monde extérieur et sacralisent la perception de l'instant.

Dans ce contexte la lecture des journaux rédigés à la fin des années soixante-dix, Le Poids du monde (novembre 1975-mars 1978) ${ }^{36}$, et au milieu de la décennie suivante, Le Matin, à la fenêtre sur le rocher (1982-1987) ${ }^{37}$, ne doit pas être négligée. Ces recueils enregistrent certes les turbulences de l'intériorité, les accès d'angoisse, les humeurs, les attentes et le vécu journalier de l'homme, mais ils brossent aussi un portrait éclaté d'un poète collectionneur de traces et d'images, observateur infatigable du monde des hommes et de la nature. Pas plus que l'Essai sur le juke-box, ils ne témoignent d'une emprise croissante de la musique dans la vie et les centres d'intérêt de leur auteur, mais confirment en filigrane certaines observations liées à La Femme gauchère. Ils manifestent une orientation nouvelle des rapports entretenus avec le phénomène musical qui dépasse désormais l'horizon culturel américain et anglo-saxon.

Les notations relatives à la musique n'abondent pas, mais leur diversité et leur affleurement insistant expriment ce que les textes considérés jusqu'ici ne laissent guère deviner : la musique est source d'une interrogation constante pour celui qui s'adonne inlassablement à l'observation des frémissements de l'âme et du monde. Il sait que la vigilance est requise, que la musique est une ingérence intolérable (GW, 277 ; Ffm, 35) que les dérives menacent les compositions symphoniques qui courent le risque de se voir gagner par la cadence de la marche (GW, 13 ; Ffm, 227), c'est-à-dire de se muer en une maléfique mise au pas comme l'expose P. Guignard. Mais il sait également que les instants de plénitude font surgir chez l'individu une musique intérieure (GW, 147, 217) et que la maison silencieuse se remplit de musique quand un être aimé en franchit le seuil (Ffm, 255, 257). Il n'ignore pas davantage qu'il existe aussi des chansons à succès qui donnent de la légèreté à l'être. 
L'écoute de l'écrivain Peter Handke peut être qualifiée d'analytique, mais pas à la façon des musicologues. Son écoute passe par le filtre d'une imagination qui saisit les correspondances et qui ouvre à l'écrivain la voie de cette langue parlée par la nature dans laquelle comme l'a dit Baudelaire "Les parfums, les couleurs et les sons se répondent ». Pour le poète épris de transparence et tendu vers une écriture qui soit un chant du monde, la musique est une clé mystérieuse. Désormais c'est la musique de Mozart qui fait vibrer la sensibilité de l'« ermite du Mönchsberg " (Ffm, 400, 513, 538). Elle ne ressortit pas à celles qui refoulent le silence ( $\mathrm{Ffm}, 502)$ et amoindrissent, anesthésient la présence au monde (Ffm, 88). L'auteur du journal, auditeur d'un concert symphonique de musique de Mozart, perçoit dans le jeu instrumental la voix idéale, la voix de la parole humaine sublimée. Cette expérience auditive rare se double d'une superposition d'images associant la perception visuelle simultanée de l'orchestre et un tableau de Giotto où la narration ne s'opère que par l'alchimie mystérieuse des attitudes, des couleurs et des sons (Ffm, 513). Une distinction doit être relevée : si les œuvres achevées citées dans la présente étude mettent pudiquement l'accent sur la dimension existentielle prise par la musique populaire venue des communautés anglophones dans le cadre de la quête identitaire de leur auteur, les Journaux tenus dans les deux décennies précédant l'Essai sur le juke-box se font quant à eux l'écho d'une interrogation profonde qui nourrit une réflexion poétique dont les acquis ne se déploieront pleinement que dans des œuvres ultérieures. Un passage de Don Juan (raconté par lui-même) (2004) peut servir d'illustration convaincante. Il fournit l'exemple d'une écriture tissée par un réseau de correspondances subtiles et qui vise à reconstruire l'unité cachée derrière les composantes disparates du monde sensible. La révélation des vibrations $\mathrm{du}$ monde et le prodige de la rencontre amoureuse s'accomplissent dans le déchaînement d'une tempête de sable à Damas. Cette symphonie des confins désertiques donne lieu à l'écriture d'une véritable partition poétique de l'instant, et elle s'achève dans un jeu éclatant de couleurs (DJ, 98-102). Cela ne signifie pas que le regard de Peter Handke s'abîme désormais dans la contemplation de contrées imaginaires, il reste le guetteur des petits faits apparemment insignifiants du quotidien. L'observation lapidaire du 28 janvier 1986 est inscrite dans la durée :

La mélodie des lumières qui passent, des trains, des voitures, des avions dans le

ciel : ma patrie ${ }^{38}$.

\section{NOTES}

1. «Wie war es mit den Liedern der Beatles, der Rolling Stones, der Creedence Clearwater Revival ? Sie haben mich 'gehoben', wie man einen Schatz hebt ».

2. Peter Handke, Versuch über die Jukebox, Frankfurt/Main, Suhrkamp Verlag, 1990. (Essai sur le juke-box). Abréviation : JB.

3. Versuch über die Müdigkeit, Frankfurt/Main, Suhrkamp Verlag, 1989. (Essai sur la fatigue). Versuch über den geglückten Tag, Frankfurt/Main, Suhrkamp Verlag, 1991. (Essai sur la journée réussie).

4. L'interview est publiée dans le Nouvel Observateur du 18 avril 1991.

5. Ich bin ein Bewohner des Elfenbeinturms, Frankfurt/Main, Suhrkamp Verlag, 1972. 
6. Ce type de paysage peut être rapproché de celui du Karst qui constitue la toile de fond de Die Wiederholung, récit d'un voyage initiatique et de la naissance du jeune homme à la poésie. Die Wiederholung, Frankfurt/Main, Suhrkamp Verlag, 1986. (Le Recommencement).

7. Uwe C. Steiner : «Das Glück der Schrift. Das graphisch-graphematische Gedächtnis in Peter Handkes Texten: Goethe, Keller, Kleist ('Langsame Heimkehr', 'Versuch über die Jukebox', 'Versuch über den geglückten Tag') », in : Deutsche Vierteljahresschrift, 1996, Heft 2, S. 256-289.

Voir aussi l'étude de Klaus Modick : «Inbilder. Kleiner Versuch über Peter Handkes Versuche » in : Merkur, 1993, Heft 4, S. 332-339.

8. Essai sur la fatigue, Essai sur le juke-box, Essai sur la journée réussie, Paris, Gallimard, collection Folio, 1998, Trad. G.-A. Goldschmidt, p. 67-68.

«In dem Asphalt des Bahnsteigs war ein großer Rußfleck; [...] dick war die schwarze Schicht, die gekreuzt wurde von den Spuren vieler verschiedener Schuhsohlen und Kofferräder » (JB, 8). [...] « Die Abdrücke dieser vielfältigen Schuhsohlen da, [...] waren ein Bild, das er mitnehmen konnte auf die Weiterfahrt » (JB, 9).

9. Die Angst des Tormanns beim Elfmeter, Frankfurt/Main, Suhrkamp Verlag, 1970. (L'angoisse du gardien de but au moment du penalty). Abréviation : AdT.

Der kurze Brief zum langen Abschied, Frankfurt/Main, Suhrkamp Verlag, 1972. (Édition de référence : suhrkamp taschenbuch, 91981). (La courte lettre pour un long adieu). Abréviation : $\mathrm{kB}$.

10. Le terme donne son titre à l'ouvrage publié en 1986. Die Wiederholung. (Le Recommencement).

11. Le propos est repris et reformulé ultérieurement (JB, 67).

12. Adt $(12,105)$, JB $(92,94)$.

13. Une expérience similaire est décrite dans la première partie de La courte lettre pour un long adieu $(\mathrm{kB}, 19)$. L'audition de la chanson d'Otis Redding, On The Dock of The Bay, à Providence peu après son arrivée aux États-Unis, fait surgir le personnage du Grand Gatsby et fait acquérir au narrateur la conviction que le changement est possible et qu'il deviendra un homme nouveau libéré de la peur.

14. On apprend bientôt qu'il s'agit de la découverte des Beatles. Le titre de la chanson des Beatles qui libère des entraves des pesanteurs intérieures est précisé ultérieurement (JB, 89). Il constitue en outre le deuxième exergue de l'essai : «And I saw her standing there » (Lennon/Mc Cartney).

15. «... scholl von dort aus der Tiefe eine Musik, bei der er zum ersten Mal im Leben, und später nur noch in den Augenblicken der Liebe, das erfuhr, was in der Fachsprache'Levitation'heißt, und das er selber mehr als ein Vierteljahrhundert später wie nennen sollte: 'Auffahrt'?, 'Entgrenzung'?, 'Weltwerdung' ?, Oder so : 'Das - dieses Lied, dieser Klang - bin jetzt ich ; mit diesen Stimmen, diesen Harmonien bin ich, wie noch nie im Leben, der geworden, der ich bin' » (JB, 87-88). (Trad. G.-A. Goldschmidt, p. 112).

16. Wunschloses Unglück, Salzburg, Residenz Verlag, 1972. (Édition de référence: suhrkamp taschenbuch, 1974). Abréviation : WU.

17. Cecile Cazort Zorach, «Freedom and remembrance : the language and biography in Peter Handke's 'Wunschloses Unglück' », in : German Quaterly, 1979, p. 486-502.

18. «Oft habe ich bei der Arbeit an der Geschichte gespürt, daß es den Ereignissen besser entsprechen würde, Musik zu schreiben, Sweet New England... » (WU, 103).

19. Peter Handke, «Die privaten Weltkriege der Patricia Highsmith», in : Der Spiegel, 29/1976, S. 89.

20. Wim Wenders, à propos de son film The Soul of a Man, produit par Martin Scorsese, in : «Terminus Memphis », Télérama, 4 août 2004, n²847.

21. Télérama, Ibid., Jim Dickinson accompagna au piano les Rolling Stones et Bob Dylan. Il a travaillé avec Wim Wenders sur la musique de Paris, Texas.

22. La courte lettre pour un long adieu, Paris, Gallimard, collection Folio, 1976, Trad. G.-A. Goldschmidt, p. 60-61 (traduction légèrement modifiée).

«'He, Mutter, warum hat denn die Idiotin so weiche Hände? Und diese Idiotin war meine 
Mutter!' schrie der Sänger. Er hob die Gitarre, kauerte sich zusammen und fing in langen, nachzitternden Akkorden zu spielen an ».

23. «... während die Musik schärfer und ungeduldiger wurde... ».

« und doch betrachtete ich ihn mit Achtung und Zuneigung. Seine Verzückung war nicht künstlich sondern sie hatte ihn überrascht, und er wußte noch nicht wohin mit ihr. Er konnte nicht mehr reden, nicht einmal stammeln, und so versuchte er sich zu erlösen, indem er sich gebärdete, als sterbe in ihm ein vorzeitliches Ungeheuer » $(\mathrm{kB}, 63-64)$.

24. La mention fugitive du groupe Creedence Clearwater Revival apparaît successivement dans $L a$ Courte lettre... (kB, 154), l'Essai sur le juke-box $(\mathrm{JB}, 96)$ et Le Matin, à la fenêtre sur le rocher (Ffm, 99, 103, 274, 533).

25. Les propos tenus par un professeur des Petits chanteurs de Vienne sont à cet égard éclairants : même adultes ils se révèlent incapables de se comporter de façon autonome (WU, 94).

26. Pascal Quignard, La haine de la musique, Paris, Calmann-Lévy, 1996, p. 221, 225-226, 239.

27. Die linkshändige Frau, Frankfurt/Main, Suhrkamp Verlag, 1976. (Édition de référence: suhrkamp taschenbuch, 1981). Abréviation : IF.

28. C'est Manfred Durzak qui élucide le mystère de l'origine de la chanson mentionnée dans le récit, jusque-là reçue comme ressortissant à la fiction.

Manfred Durzak, Peter Handke und die deutsche Gegenwartsliteratur : Narziß auf Abwegen, Stuttgart, Kohlhammer, 1982, S. 145.

29. Russel E. Brown, «The Left Handed Women of Peter Handke and Jimmy Reed ", in : modern fiction studies, 1990, vol. 36, n³, p. 375-401.

30. Don Juan (erzählt von ihm selbst), Frankfurt/Main, Suhrkamp Verlag, 2004. Abréviation : DJ.

31. La femme gauchère, Paris, Gallimard, collection Folio, 1978, Trad. G.-A. Goldschmidt, p. 91.

« Ich möchte dich IN EINEM FREMDEN ERDTEIL sehen

Denn da werde ich dich unter den andern endlich allein sehen

Und du wirst unter tausend andern MICH sehen

Und wir werden endlich aufeinander zugehen » (lF, 102).

32. Interview de P. Handke accordée au journal Le Monde, 18 mai 1978.

33. Le passage de trains est un élément sonore et visuel récurrent dans le récit.

34. Le soir de la rupture du couple, une boîte à musique, dans la chambre de l'enfant où Marianne viendra chercher refuge, égrène elle aussi La lettre à Élise. Une notation dans le Journal Le poids du monde, ouvre la voie à l'interprétation : « les lamentables pianos dans les appartements : culs-de$\mathrm{sac} »(\mathrm{GW}, 161)$.

35. La métamorphose opérée au cours d'une soirée improvisée, réunissant tous les personnages chez Marianne, a changé de dimension: rien de ne subsiste du fond musical et de la danse caractérisant la scène du récit.

36. Das Gewicht der Welt, Salzburg, Residenz Verlag, 1977. (Édition de référence: suhrkamp taschenbuch, 21982). (Le Poids du monde). Abréviation : GW.

37. Am Felsfenster morgens, Salzburg, Residenz Verlag, 1998. (Le Matin, à la fenêtre sur le rocher). Abréviation : Ffm.

38. « Die Melodie der wandernden Lichter, der Züge, der Autos, der Flugzeuge im Himmel : mein Vaterland » (Ffm, 340). 


\section{RÉSUMÉS}

Il n'est guère question de musique dans la prose handkéenne. Pourtant la rétrospective des années de jeunesse et d'études sur laquelle s'appuie l'Essai sur le juke-box (1990) invite à s'intéresser aux traces laissées par la musique dans les récits des années soixante-dix abordant le thème de la quête de l'identité. Il ressort d'une lecture croisée de l'essai et de ces oeuvres que la musique populaire vocale enracinée dans les espaces américain et anglo-saxon a joué un rôle de premier plan en raison de sa dimension de communication verbale. Elle a exercé une influence clarificatrice et émancipatrice sur le jeune Handke. La femme gauchère quant à elle fait apparaître au travers de ses deux versions bien distinctes (récit et film) l'importance que revêt la musique pour l'écrivain. Quelques notations disséminées dans les Journaux écrits entre 1975 et 1987 mettent par ailleurs en évidence que la réflexion sur le mystère de la musique en général, et sur celle des dix-septième et dix-huitième siècles en particulier, constitue l'un des fondements de la poétique de $\mathrm{P}$. Handke, contemplateur et chantre des frémissements de l'âme et du monde.

Musik kommt in P. Handkes Prosawerk selten vor, jedoch gibt der Versuch über die Jukebox (1990), der auf die Jugend und die Studentenzeit des Dichters zurückblickt, Anlass zu einer Untersuchung der Spuren, die die Musik in den Erzählungen der siebziger Jahre hinterlassen hat, in denen die Identitätssuche thematisiert wird. Vom Versuch über die Jukebox ausgehend, zeigt der vorliegende Aufsatz, dass Folksong und Blues als vokale, erzählende, kommunikationsträchtige Musikformen einen entscheidenden Einfluss auf den jungen Handke ausübten, da sie ihm dazu verhalfen, über sich klarer zu werden. Darüber hinaus zeigt eine vergleichende Analyse der zwei stark voneinander abweichenden Fassungen von Die linkshändige Frau (als Buch und Film) zum ersten Mal Handkes Auseinandersetzung mit der Musik auf. Einige Aufzeichnungen aus den Tagebüchern, die zwischen 1975 und 1987 entstanden sind, belegen eindeutig, dass für P. Handke, der den Leser zum Mitschauen, Mitdenken, Mitentdecken einlädt, das Reflektieren über das Rätsel der Musik - nicht zuletzt über die der vergangenen Jahrhunderte - ein wesentlicher Beitrag zu seiner Poetik ist.

\section{AUTEUR}

\section{BRIGITTE DESBRIÈRE-NICOLAS}

Université Charles-de-Gaulle - Lille 3 